Article

\title{
Thermal Properties of Binary Filler Hybrid Composite with Graphene Oxide and Pyrolyzed Silicon-Coated Boron Nitride
}

\author{
Jaehyun Wie and Jooheon Kim * (D) \\ School of Chemical Engineering and Materials Science, Chung-Ang University, Seoul 156-756, Korea; \\ grwie23@naver.com \\ * Correspondence: jooheonkim@cau.ac.kr
}

Received: 8 October 2020; Accepted: 28 October 2020; Published: 30 October 2020

check for updates

\begin{abstract}
To improve the thermal conductivity of a composite material, the filler dispersion and the interfacial adhesion between the filler and the matrix are important factors. A number of methods for satisfying these criteria are presented herein. Thus, graphene oxide (GO) is incorporated to enhance the dispersion state of surface-modified boron nitride (BN) by increasing the viscosity of the epoxy matrix and by providing steric hindrance. Meanwhile, polysilazane (PSZ) coating and thermolysis were used to enhance the wettability by providing structural similarity between the coating material and the epoxy matrix. Due to these strategies, the thermal conductivity was improved by $253 \%$ compared to that of the neat epoxy at a filler fraction of $40 \mathrm{wt} \%$.
\end{abstract}

Keywords: polymer composite; thermal conductivity; surface modification

\section{Introduction}

Since the efficient dissipation of accumulated heat is an important factor for the credibility and durability of electronic devices, thermal management has been an issue of great interest for many decades $[1,2]$. In this respect, the dielectric properties and excellent processability of polymers make these a popular choice for use as thermal interface materials (TIMs) [3]. However, the practical applicability of polymers is limited by their generally very low thermal conductivities $\left(\sim 0.2 \mathrm{~W} \cdot \mathrm{m}^{-1} \cdot \mathrm{k}^{-1}\right)$. To address this problem, various kinds of thermally conductive fillers have been used, including carbon-based, ceramic, and metal fillers. Boron nitride (BN) is generally used as a ceramic filler to improve the thermal conductivity and provide electrical insulation $[4,5]$.

To improve the thermal conductivity, the filler dispersion and the interfacial adhesion in the composite are important issues [6,7]. Fillers tend to aggregate due to Van der Waals forces, thus leading to uneven dispersion in the composite [8]. This, in turn, affects the efficiency of the connecting networks for electrical and thermal transfer in the composite [9]. Meanwhile, structural incompatibility between rigid inorganic thermally conductive fillers such as $\mathrm{BN}$ and the polymer matrix often results in poor interfacial adhesion [10]. This causes phonon scattering and hinders phonon transfer by creating thermal boundary resistance. To address this problem, many researchers have tried various surface modifications for improving the affinity between the filler and the matrix [11-13].

Silicon-based materials such as polysilazane (PSZ) are used to coat substrates in order to provide chemical resistance and high thermal stability [14]. In addition, thermolysis is used to convert the PSZ into a ceramic [15]. The resulting structure has been shown to vary depending on the temperature of thermolysis, with the primary chain being converted from $\mathrm{Si}-\mathrm{N}$ to $\mathrm{Si}-\mathrm{O}$ at $1000{ }^{\circ} \mathrm{C}$ [16].

In the present study, several methods are used to address the various above-mentioned problems. The incorporation of graphene oxide $(\mathrm{GO})$ is found to improve the filler dispersion due to the increased 
viscosity of the matrix and the steric hindrance caused by the GO. In addition, PSZ coating and thermolysis are used to cover the surface of the BN with siloxane groups and, thus, enhance the interfacial adhesion between the BN and the epoxy-terminated dimethylsiloxane (ETDS) matrix. Due to the heat transfer route provided by the enhanced dispersion and interfacial adhesion between the filler and the matrix, the obtained GO/P-BN/epoxy composite exhibited an improved thermal conductivity of $0.4517 \mathrm{~W} / \mathrm{m}^{-1} \cdot \mathrm{K}^{-1}$, which is a $253 \%$ enhancement over that of the neat epoxy. Hence, this facile strategy was successfully used to improve the thermal conductivity of the polymer composite.

Fillers used in this paper were named as an abbreviated form such as R-BN (raw BN) and P-BN (polysilazane coated BN).

\section{Experimental}

\subsection{Materials}

Natural universal grade graphite flakes ( 200 mesh, 99.9995\% (metals basis)), and concentrated hydrogen peroxide $\left(\mathrm{H}_{2} \mathrm{O}_{2}, 27 \%\right.$ w/w aq.) were purchased from Alfa Aesar (St. Louise, MO, USA). Sulfuric acid $\left(\mathrm{H}_{2} \mathrm{SO}_{4}, 95 \%\right.$ aq.), phosphoric acid $\left(\mathrm{H}_{3} \mathrm{PO}_{4}, 80 \%\right.$ aq. $)$, hydrochloric acid $(25 \%, 20 \%$ aq.) were obtained from DaeJung Chemicals Co., Seoul, Korea. Potassium permanganate $\left(\mathrm{KMnO}_{4}, 99 \%\right)$ and 4-4'-diaminodiphenylmethane (97\%) were purchased from Sigma Aldrich (Seoul, Korea). Polysilazane (KiON HTT 1800) was supplied by Clariant GmbH (Muttenz, Switzer land). Epoxy resin (KF105; ETDS) was obtained from Shin-Etsu Silicon (Seoul, Korea). Boron nitride was obtained from LG Innotek (Seoul, Korea).

\subsection{Preparation of Graphene Oxide (GO)}

The $\mathrm{GO}$ was prepared from graphite flakes via an improved Hummers' method [17]. Graphite (3 g) was first added to a 9:1 mixture of $\mathrm{H}_{2} \mathrm{SO}_{4} / \mathrm{H}_{3} \mathrm{PO}_{4}(400 \mathrm{~mL})$ and the mixture was stirred for $4 \mathrm{~h}$ in an ice bath to produce a homogeneous dispersion. The $\mathrm{KMnO}_{4}(18 \mathrm{~g})$ was then slowly introduced into the mixture and the reaction was stirred for $12 \mathrm{~h}$ at $323 \mathrm{~K}$. After reaction, the mixture was cooled to room temperature (RT) and transferred to a solution of $30 \% \mathrm{H}_{2} \mathrm{O}_{2}(5 \mathrm{~mL})$ and $400 \mathrm{~mL}$ frozen de-ionized (DI) water. Finally, the mixture was centrifuged with $\mathrm{HCl}(400 \mathrm{~mL})$ and purified with DI water. The resulting suspension was poured onto a glass slide and dried at $323 \mathrm{~K}$ for $72 \mathrm{~h}$ to yield the $\mathrm{GO}$ as a solid.

\subsection{Surface-Modification of Boron Nitride (BN)}

The BN (10g) and PSZ (1g) were stirred in acetone for $6 \mathrm{~h}$ at room temperature. The mixture was then washed twice with acetone before placing the precipitate in a convection oven at $323 \mathrm{~K}$ overnight. Finally, the dried powder was heated to $433 \mathrm{~K}$ for $4 \mathrm{~h}$ for the crosslinking reaction to occur, which was followed by pyrolysis at $1273 \mathrm{~K}$ for $2 \mathrm{~h}$ under an argon atmosphere.

\subsection{Fabrication of the GO/P-BN/Epoxy Composite}

The GO was dispersed in ethanol using an ultrasonic bath for $1 \mathrm{~h}$. Meanwhile, the ETDS and 4-4'-diaminodiphenylmethane curing agent (weight ratio of 5:1) were stirred on a hotplate at $403 \mathrm{~K}$ for $1 \mathrm{~h}$, followed by addition of the P-BN. The solution was maintained at this temperature under stirring until all traces of the solvent were removed; then, the obtained mixture was poured onto a Teflon plate and transferred to a vacuum oven at $353 \mathrm{~K}$ for $30 \mathrm{~min}$ to remove any air bubbles. The doctor blade technique was then used to control the uniformity of the film thickness. Finally, the composite film was cured in a furnace at $453 \mathrm{~K}$ for $1 \mathrm{~h}$. The fabricated composites are denoted GO/P-BN/epoxy.

\section{Results and Discussion}

The IR spectra of the R-BN and P-BN in Figure 1a both display the typical peaks of oxygen-containing groups, including those of $\mathrm{OH}$ at $\sim 3400 \mathrm{~cm}^{-1}$, the $\mathrm{C}=\mathrm{O}$ stretching at $1750 \mathrm{~cm}^{-1}$, and the sp $\mathrm{sp}^{2}$-hybridized carbon group stretching at $1620 \mathrm{~cm}^{-1}$ [18]. In addition, the peaks at $820 \mathrm{~cm}^{-1}$ 
(out-of-plane B-N-B bending vibration) and $1360 \mathrm{~cm}^{-1}$ (in-plane B-N stretching vibration) are intrinsic to the $\mathrm{BN}$ [19]. Compared to the R-BN, the spectrum of the P-BN displays additional peaks due to the $\mathrm{Si}-\mathrm{CH}_{3}\left(1250 \mathrm{~cm}^{-1}\right)$, and $\mathrm{Si}-\mathrm{O}-\mathrm{Si}\left(\sim 1030 \mathrm{~cm}^{-1}\right)$ derived from the PSZ coating and thermolysis [20].

(a)

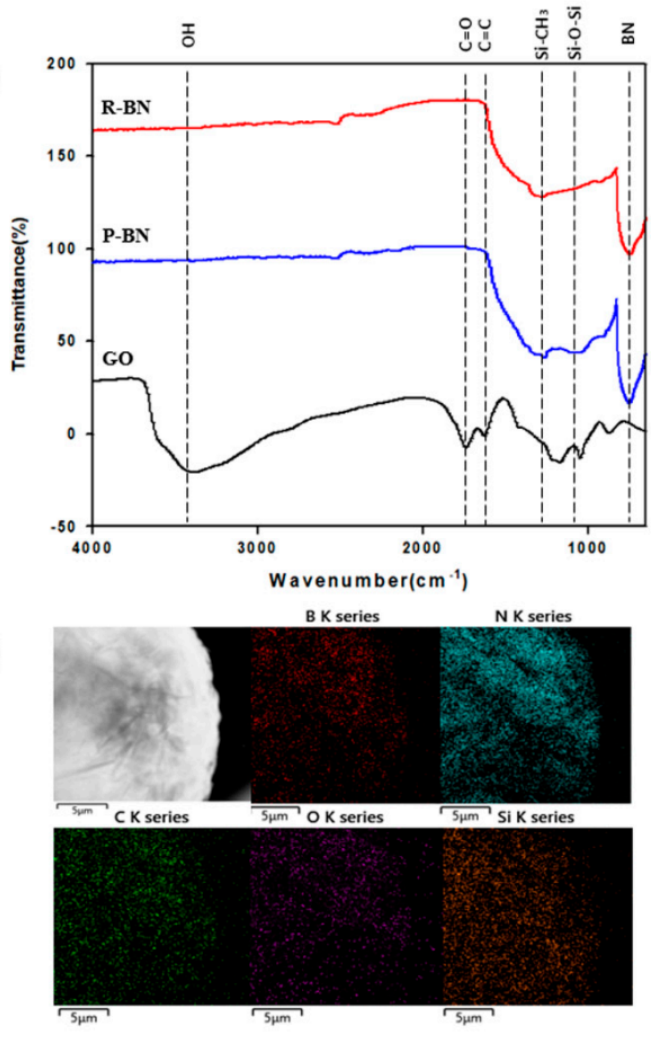

(b)

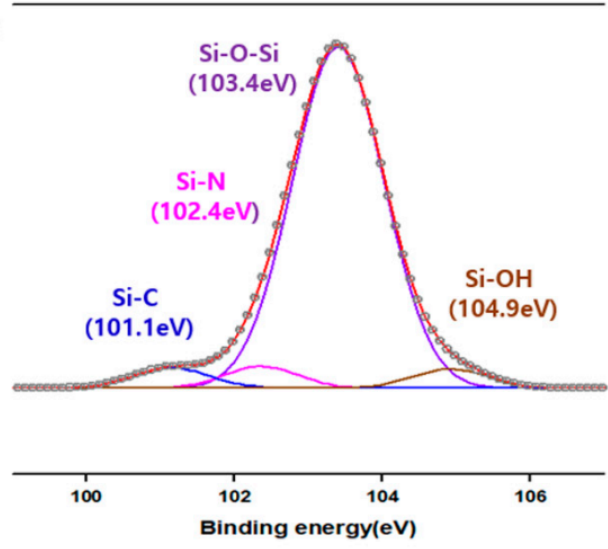

(d)

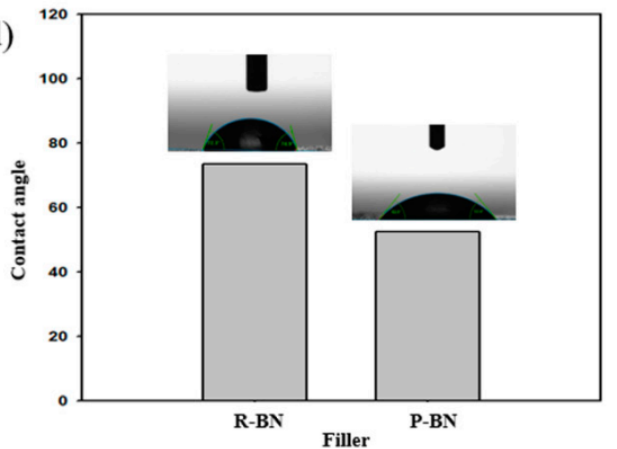

Figure 1. Confirmation of filler surface modification. (a) FT-IR spectra of fillers, (b) XPS Si2p deconvolution data of P-BN, (c) TEM-EDS image of P-BN, (d) ETDS contact angle of fillers.

The XPS analysis of the P-BN is shown in Figure 1b. The peak-fitting performed using the Person7 peak shape software package reveals one large peak and three minor peaks. The primary peak at $103.4 \mathrm{eV}$ is attributed to the $\mathrm{Si}-\mathrm{O}-\mathrm{Si}$ bonds, thus demonstrating the successful ceramization of PSZ by calcination at $1000{ }^{\circ} \mathrm{C}$. The two minor peaks at 101.1 and $102.4 \mathrm{eV}$ are attributed to the $\mathrm{Si}-\mathrm{C}$ and $\mathrm{Si}-\mathrm{N}$, respectively, which comprise the main chain of the raw PSZ. The remaining minor peak at $104.9 \mathrm{eV}$ represents the Si-OH that was formed by hydrolysis of the raw PSZ [16,21]. A comparison of the peak intensities indicates that the coating material consists primarily of $\mathrm{Si}-\mathrm{O}-\mathrm{Si}$ (siloxane) bonds, which are also present in the ETDS.

To investigate the elemental composition of the P-BN, EDS analysis was performed, as shown in Figure 1c. As expected, the elements B, N, Si, C, and O were successfully detected. Moreover, the EDS mapping images demonstrate the uniform distributions of these five atoms.

To investigate the improvement in affinity between the matrix and the filler due to surface modification, the ETDS contact angle was measured. Here, the R-BN and P-BN were each compressed into pellet form via the hot press method; then, ETDS $(10 \mu \mathrm{L})$ was dropped onto the surface of each pellet. The contact angles between the ETDS and the R-BN and P-BN were $73.6^{\circ}$ and $52.6^{\circ}$, respectively. Since both the P-BN and ETDS contain siloxane groups, the improved interfacial adhesion between the filler and the matrix is attributed to their structural similarity [10].

The morphologies of the epoxy composites were investigated by cross-sectional SEM microscopy. Thus, the SEM image in Figure 2a reveals a distinctly layered morphology due to the tendency for the high-density R-BN to diffuse downwards during the curing process. This phenomenon impairs the dispersion of fillers in the matrix and adversely affects the performance of the composites. The interfacial 
adhesion between the filler and the matrix was also revealed by the SEM images, with numerous gaps being observed around the filler due to the poor wettability of the R-BN (Figure 2d). These gaps may obstruct the flow of heat and result in heat loss. However, the introduction of GO into the composite is seen to improve the level of dispersion of the filler in the matrix (Figure 2b). This may be attributed to several possible reasons. First, an increase in the viscosity of the matrix is advantageous for reducing the mobility of $\mathrm{BN}$ during the mechanical mixing and curing processes, which has previously been shown to sink in the bottom of the matrix [22]. It is well known that the addition of GO increases the viscosity of the matrix due to the high aspect ratio of GO. Secondly, steric hindrance between two types of fillers in a composite material has been shown to affect the behavior of the filler [23]. Thus, the well-dispersed GO also helps prevent the BN from diffusing downwards and improve the level of BN dispersion in the matrix. However, an examination of Figure 2e indicates that the interfacial adhesion was not greatly improved. This residual problem was addressed by the successful surface modification of the BN to provide structural similarity with the ETDS matrix, as shown in Figure $2 \mathrm{f}$. Thus, after calcination at $1000{ }^{\circ} \mathrm{C}$, both PSZ and ETDS contained siloxane bonds in the main chain. As previously reported, structural change on the surface of $\mathrm{BN}$ increases the compatibility between the filler and the matrix [10].
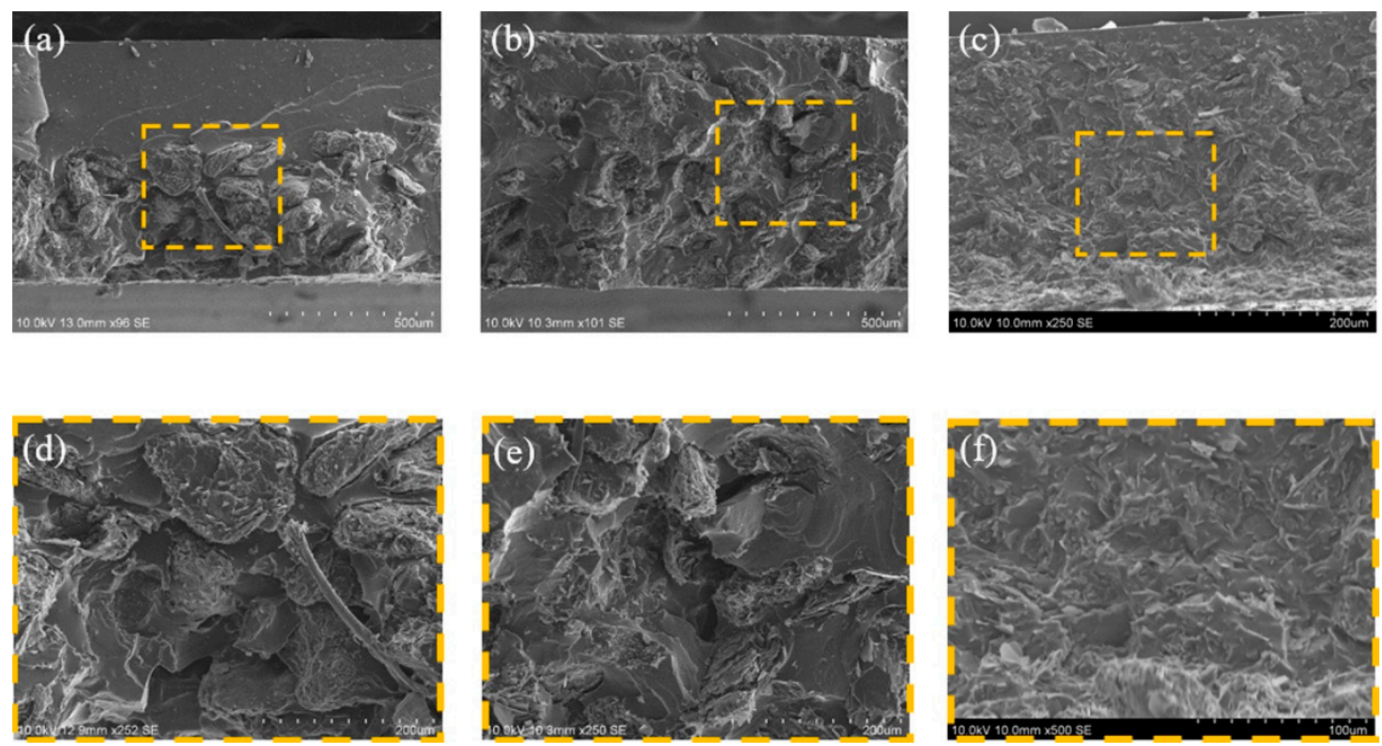

Figure 2. SEM cross section image of fabricated composites. (a) R-BN/epoxy composite, (b) GO/R-BN/ epoxy composite, (c) GO/P-BN/epoxy composite, (d) magnified area of (a) image, (e) magnified area of (b) image, (f) magnified area of (c) image.

The tensile strengths of the various samples were measured to examine the effects of GO introduction and surface modification with PSZ, and the results are presented in Figure 3. The tensile strength of the neat epoxy is seen to be $0.31 \mathrm{MPa}$, whereas that obtained after the incorporation of GO is quite different $(0.6 \mathrm{MPa})$. This can be explained by the morphology of the composite shown in the SEM cross section, where the R-BN was seen to aggregate at the bottom of the matrix, thus leading to a variation in the composition of the sample depending on the position. Hence, while the upper part of the matrix is compositionally similar to the neat epoxy, a much higher concentration of R-BN is present at the bottom of the matrix. Due to this drastic variation in the composition of the composite, the sample was broken twice during the tensile test. At the first break, the tensile strength was similar to that of the neat epoxy, while a decrease in tensile strength was observed at the second break. However, after GO introduction, the tensile strength of the BN/GO/epoxy composite was improved by 93\% compared to that of the neat epoxy due to the enhanced dispersion of the filler in the presence of GO. Thus, the tensile strength of the composite is determined by the level of dispersion of the filler along the applied load direction [24]. When the filler is well dispersed in the matrix, the area over 
which load can be transferred is increased compared to that in the R-BN/epoxy composite. As the load is applied to the composite, cracks and voids therein begin to propagate and, when a crack approaches the filler, the stress dramatically increases in intensity until filler-matrix debonding occurs. In the case of the P-BN/GO/epoxy composite, the interfacial adhesion between the filler and the matrix was improved, as shown in the SEM cross-sectional image, so that the filler-matrix debonding was less likely after surface modification. This resulted in a $13 \%$ increase in the tensile strength due specifically to the PSZ coating. Moreover, this improvement in tensile strength is seen to increase with increasing filler concentration up to $30 \mathrm{wt} \%$, although a decrease in the tensile strength is observed at a filler concentration of $40 \mathrm{wt} \%$. This can be attributed to the interruption of the crosslinking between the epoxy and the matrix in the presence of excess filler.
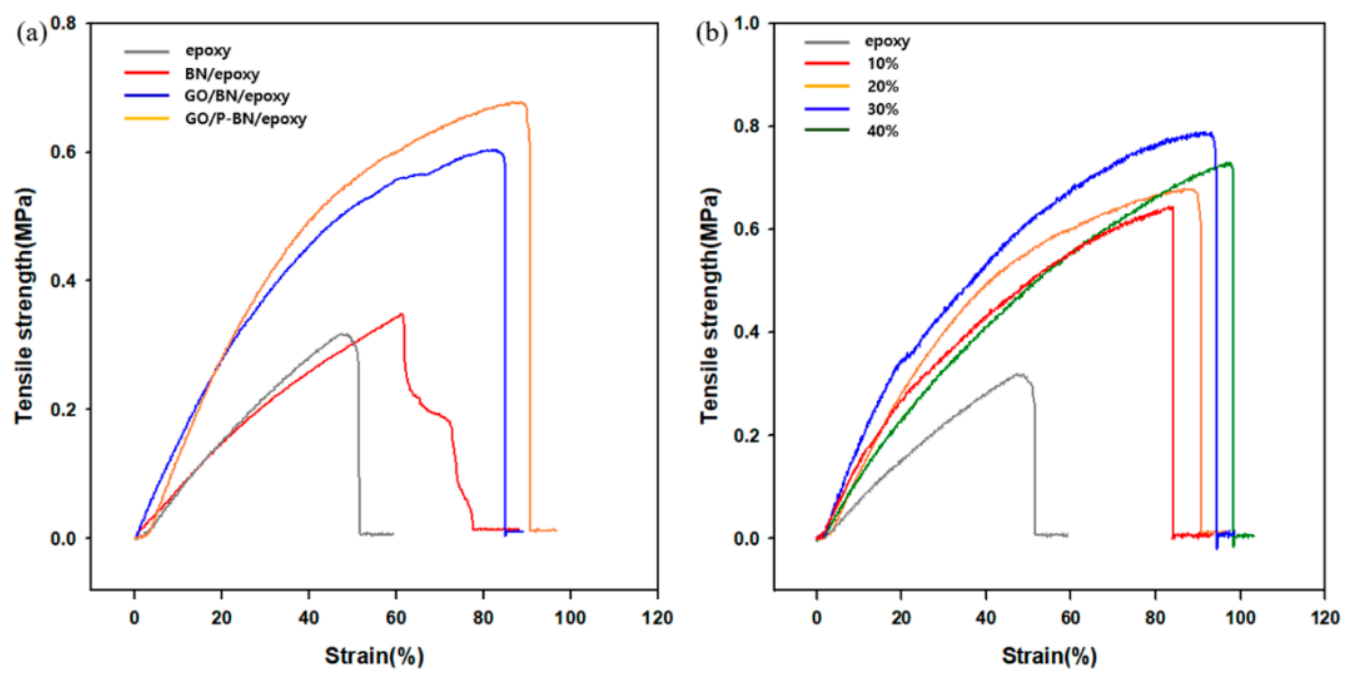

Figure 3. Mechanical property measured by UTM. (a) tensile strength measured for confirming the effect of surface modification and GO incorporation; (b) tensile strength measured for confirming the effect of P-BN weight fraction in a GO/P-BN/epoxy composite.

The fact that intrinsic thermal conductivity of GO is very low $\left(0.5-1.0 \mathrm{~W} \cdot \mathrm{m}^{-1} \cdot \mathrm{K}^{-1}\right)$ at room temperature was already well known in prior literature [25], which was also confirmed from Figure $4 \mathrm{~b}$. Similar thermal conductivities were achieved for composites incorporating GO fractions greater than $0.5 \mathrm{wt} \%$ as shown in Figure 4a. Based on these data, the optimum ratio of BN to GO was found to be 40:1 for both the GO/R-BN/epoxy and GO/P-BN/epoxy composites. With further increase in the BN fraction, the weight of GO incorporation also increased, but the thermal conductivity was not affected, as shown in Figure 4b.
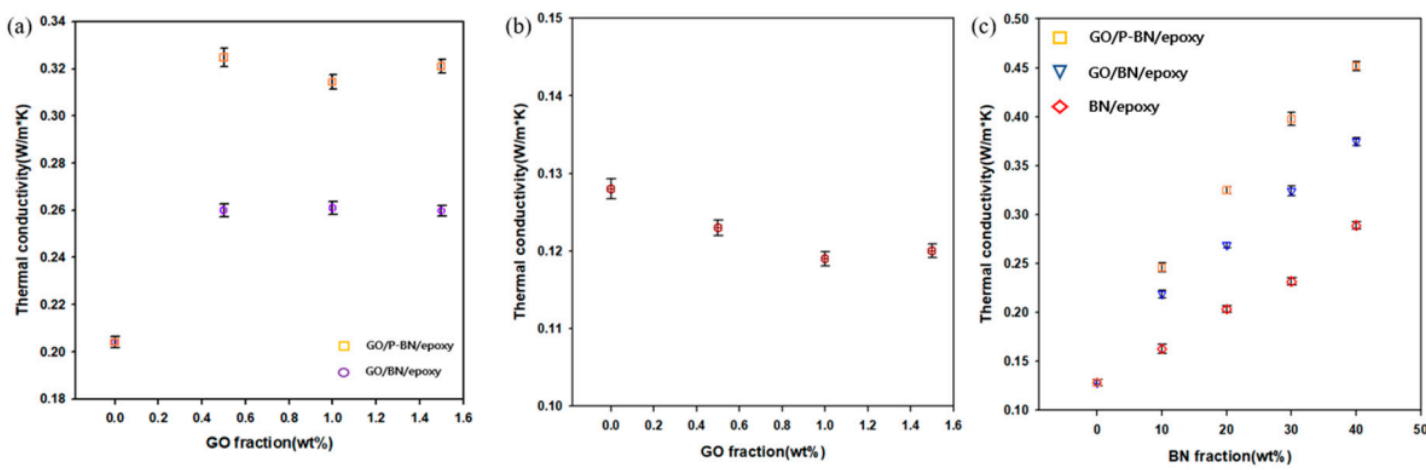

Figure 4. Thermal conductivity data of various samples. (a) thermal conductivity of GO/R-BN/epoxy composite depending on the GO weight fraction; (b) thermal conductivity of GO/epoxy composite depending on the GO weight fraction; (c) thermal conductivity of fabricated composites in this research. 
To investigate the effects of surface modification and GO incorporation, the thermal conductivities of the composites were calculated and the results are presented in Figure 4c. In BN/ETDS composites, BN only existed at the bottom of the matrix, which cannot form a heat transfer path. As filler fraction increased, the filler occupied more area in the matrix and increased thermal conductivity. Furthermore, with the incorporation of $\mathrm{GO}$, the $\mathrm{BN}$ is no longer aggregated at the bottom of the matrix, but, rather, becomes well dispersed throughout, so that the thermal conductivity of the GO/BN/epoxy composite is increased compared to that of the BN/epoxy composite. Moreover, the effect of surface modification is observed in the GO/P-BN/epoxy composite as the enhanced affinity between the filler and the matrix readily fill up any voids. To investigate the effect of the interface on the thermal conductivity, the void fractions of the composites were calculated from the theoretical and measured densities in accordance with the following equations:

$$
\begin{aligned}
& \text { void fraction }=\left(\rho_{\text {theoretical }}-\rho_{\text {experimental }}\right) / \rho_{\text {theoretical }} ; \\
& \text { and } \rho_{\text {theoretical }}=1 /\left[\left(W_{\text {filler }} / \rho_{\text {filler }}\right)+\left(W_{\text {matrix }} / \rho_{\text {matrix }}\right)\right],
\end{aligned}
$$

where $W_{\text {filler }}$ and $W_{\text {matrix }}$ are the weight fractions of the filler and the epoxy matrix, respectively, and $\rho_{\text {filler }}$, and $\rho_{\text {matrix }}$ are the densities of the filler and the epoxy matrix, respectively. It has previously been shown that much heat loss occurs at the voids in such composite materials due to the low thermal conductivity therein (thermal conductivity of air $=5 \times 10^{-5}$ ) [26]. As shown in Figure 5, the GO incorporation and surface modification of $\mathrm{BN}$ both act to decrease the void fraction by improving the dispersion of the filler throughout the matrix.
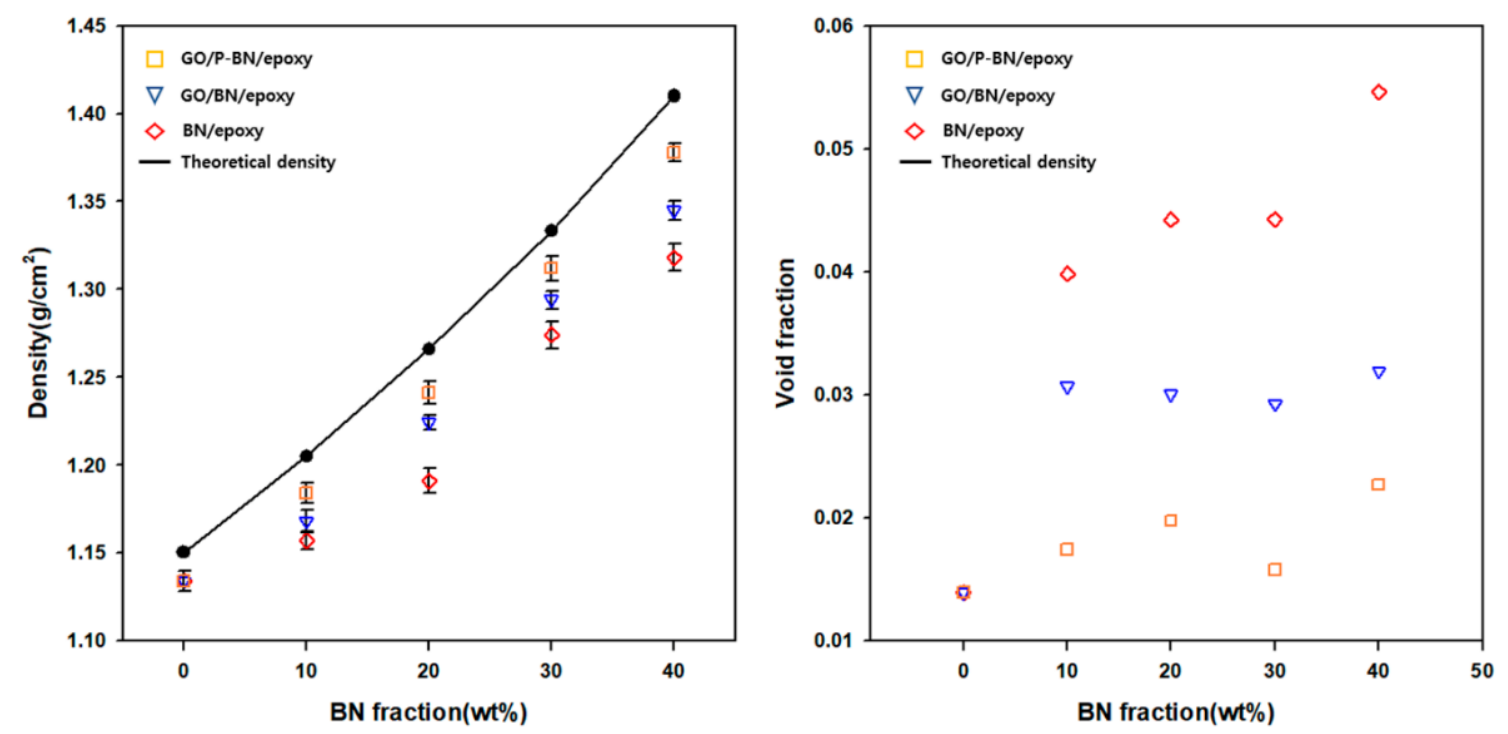

Figure 5. (a) theoretical expected densities and experimental densities; (b) void fraction of fabricated composites in this research.

The thermal conductivities of the composites were estimated using the Agari-Uno model, which is given by the following equation [27]:

$$
\log K_{\mathrm{c}}=\left[\left(V_{\mathrm{f}} C_{2}\right) \times \log K_{\mathrm{f}}\right]+\left[\left(1-V_{\mathrm{f}}\right) \times \log \left(C_{1} K_{\mathrm{m}}\right)\right]
$$

where $V_{\mathrm{f}}$ is volume fraction of fillers, $K_{\mathrm{m}}$ is the thermal conductivity of matrix, and $K_{\mathrm{c}}$ is the thermal conductivity of composites. Thus, $C_{1}$ and $C_{2}$ could be calculated from the slope and $y$-axis intercept, respectively, in Figure 6a. The values of $C_{1}$ and $C_{2}$ are generally between 0 and 1 , with a $C_{1}$ value close to 1 indicating that the filler is a suitable match for the polymer structure, and a $C_{2}$ value close to 1 indicating that the thermally conductive route is well formed [28]. In the present work, the values of $C_{1}$ 
and $C_{2}$ were found to increase after the GO incorporation and surface modification, thus demonstrating that these factors are effective for improving the thermal conductivity in agreement with previous work [29].
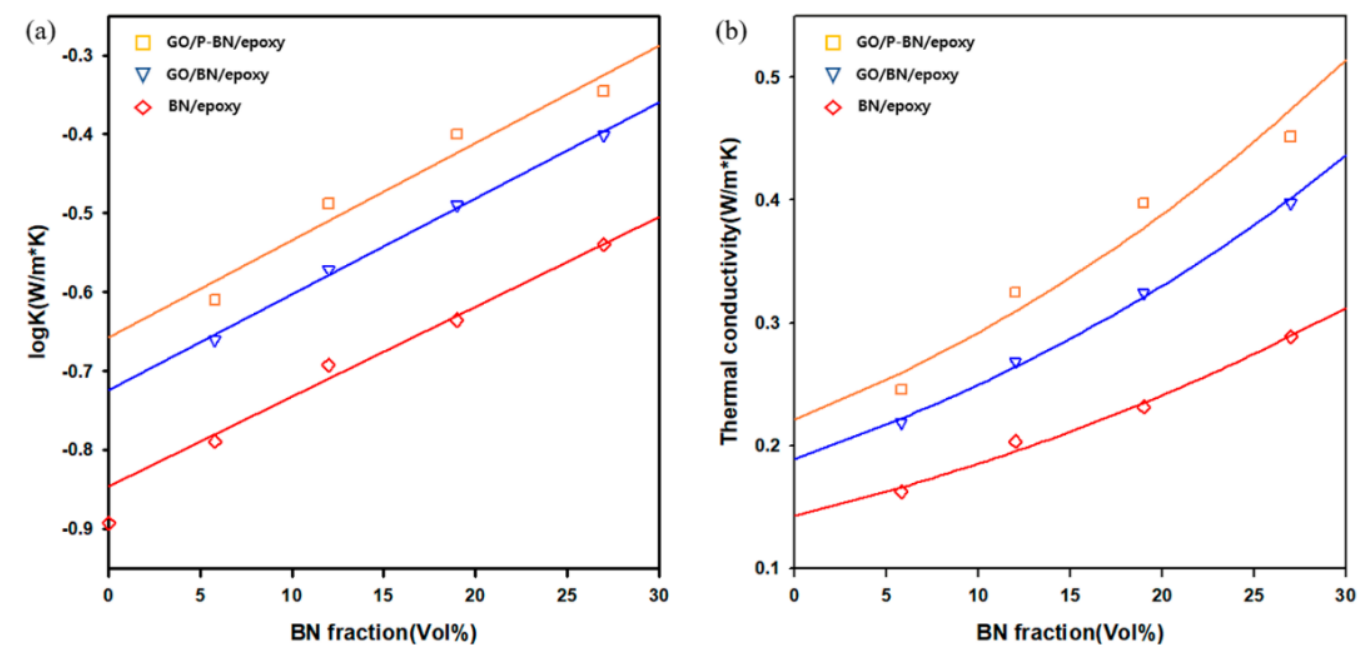

Figure 6. (a) logarithm of thermal conductivity of composites; (b) comparison of measured thermal conductivities of composites with calculated results using the Agari-Uno model.

The storage modulus provides information on the degree of elasticity and the load bearing ability of the composites [30] along with the stiffness, degree of crosslinking, and interfacial adhesion between the filler and the matrix [31]. The storage modulus of composites was measured by using the DMA graph as a function of temperature. In the present work, the storage modulus of the neat epoxy was found to be $2.2 \mathrm{GPa}$. In addition, it was continuously improved as methods in this research were applied. Thus, the efficient placement of the filler and the enhanced interfacial adhesion both increased the storage modulus as well as the thermal conductivity. However, the storage modulus was found to decrease as the temperature increased because the state of the polymer chains changed from the glass state to the rubbery state [32]. The glass transition temperature $\left(T_{\mathrm{g}}\right)$ values were obtained from Figure $7 \mathrm{~b}$. The $\tan \delta$ values display a similar tendency to the storage modulus. Overall, the dispersion of filler due to the incorporation of GO, along with the enhanced interfacial adhesion, resulted in a decreased mobility of the polymer chains and, hence, an increased glass transition temperature.
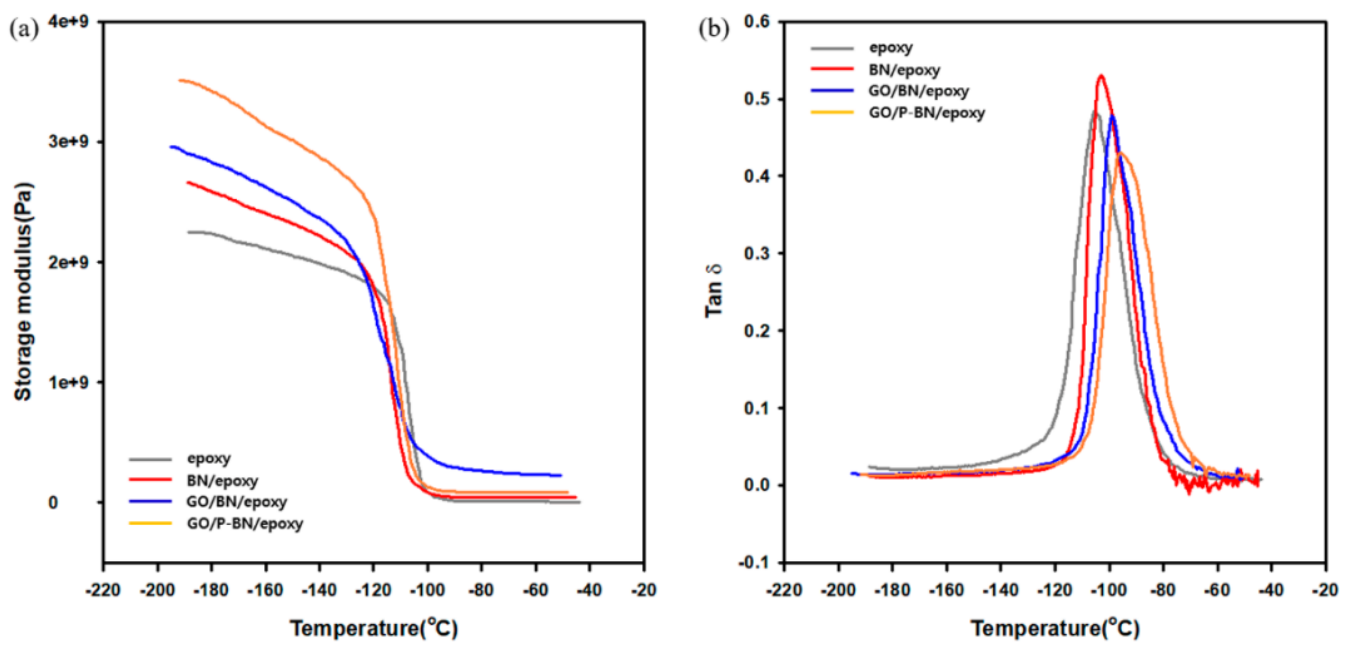

Figure 7. Mechanical property measured by DMA. (a) storage modulus of composites; (b) $\tan \delta$ of composites. 


\section{Conclusions}

In the present study, dispersive and surface modification approaches were developed in order to improve the thermal conductivity of composites. The addition of GO was shown to increase the viscosity of the epoxy matrix by providing steric bulk, thus enhancing the filler dispersion. In addition, PSZ coating and thermolysis were shown to increase the structural similarity between the surface of the filler and the epoxy matrix. Due to these effects, the thermal conductivity of the GO/P-BN/epoxy was improved by $253 \%$ compared to that of the neat epoxy. Thus, it has been established that the effect of GO incorporation is to enhance the dispersion of the filler in the matrix and, thus, enhance the performance of the polymer composite.

Author Contributions: J.W.: data curation, investigation, original draft. J.K.: funding acquisition, review \& editing, supervision, project administration. All authors have read and agreed to the published version of the manuscript.

Funding: This research was supported by the MSIT (Ministry of Science and ICT), Korea, under the ITRC (Information TechnologyResearch Center) support program (IITP-2020-2020-0-01655) supervised by the IITP (Institute of Information \& Communications Technology Planning \& Evaluation).

Conflicts of Interest: The authors declare no conflict of interest.

\section{References}

1. Leung, S.N. Thermally conductive polymer composites and nanocomposites: Processing-structure-property relationships. Compos. Part B Eng. 2018, 150, 78-92. [CrossRef]

2. Mehra, N.; Mu, L.; Ji, T.; Li, Y.; Zhu, J. Moisture driven thermal conduction in polymer and polymer blends. Compos. Sci. Technol. 2017, 151, 115-123. [CrossRef]

3. Lee, S.-Y.; Singh, P.; Mahajan, R.L. Role of oxygen functional groups for improved performance of graphene-silicone composites as a thermal interface material. Carbon 2019, 145, 131-139. [CrossRef]

4. Teng, C.; Su, L.; Chen, J.; Wang, J. Flexible, thermally conductive layered composite films from massively exfoliated boron nitride nanosheets. Compos. Part A Appl. Sci. Manuf. 2019, 124, 105498. [CrossRef]

5. Han, J.; Du, G.; Gao, W.; Bai, H. An anisotropically high thermal conductive boron nitride/epoxy composite based on nacre-mimetic 3D network. Adv. Funct. Mater. 2019, 29. [CrossRef]

6. Han, Z.; Fina, A. Thermal conductivity of carbon nanotubes and their polymer nanocomposites: A review. Prog. Polym. Sci. 2011, 36, 914-944. [CrossRef]

7. Chen, H.; Ginzburg, V.V.; Yang, J.; Yang, Y.; Liu, W.; Huang, Y.; Du, L.; Chen, B. Thermal conductivity of polymer-based composites: Fundamentals and applications. Prog. Polym. Sci. 2016, 59, 41-85. [CrossRef]

8. Yue, L.; Pircheraghi, G.; Monemian, S.A.; Manas-Zloczower, I. Epoxy composites with carbon nanotubes and graphene nanoplatelets-Dispersion and synergy effects. Carbon 2014, 78, 268-278. [CrossRef]

9. Loos, M.; Yang, J.; Feke, D.; Manas-Zloczower, I. Effect of block-copolymer dispersants on properties of carbon nanotube/epoxy systems. Compos. Sci. Technol. 2012, 72, 482-488. [CrossRef]

10. Xie, X.-L.; Tang, C.-Y.; Zhou, X.-P.; Li, R.K.-Y.; Yu, Z.-Z.; Zhang, Q.-X.; Mai, Y.-W. Enhanced interfacial adhesion between PPO and glass beads in composites by surface modification of glass beads via in situ polymerization and copolymerization. Chem. Mater. 2004, 16, 133-138. [CrossRef]

11. Jiang, Y.; Shi, X.; Feng, Y.; Li, S.; Zhou, X.; Xie, X. Enhanced thermal conductivity and ideal dielectric properties of epoxy composites containing polymer modified hexagonal boron nitride. Compos. Part A Appl. Sci. Manuf. 2018, 107, 657-664. [CrossRef]

12. Wu, X.; Yang, Z.; Kuang, W.; Tang, Z.; Guo, B. Coating polyrhodanine onto boron nitride nanosheets for thermally conductive elastomer composites. Compos. Part A Appl. Sci. Manuf. 2017, 94, 77-85. [CrossRef]

13. Kim, K.; Wie, J.; Kim, J. Synergistic interaction of P and N co-doping EDTA with controllable active EDTA-cobalt sites as efficient electrocatalyst for oxygen reduction reaction. J. Ind. Eng. Chem. 2020, 83, 252-259. [CrossRef]

14. Bazarjani, M.S.; Kleebe, H.-J.; Müller, M.M.; Fasel, C.; Yazdi, M.B.; Gurlo, A.; Riedel, R. Nanoporous silicon oxycarbonitride ceramics derived from polysilazanes in situ modified with nickel nanoparticles. Chem. Mater. 2011, 23, 4112-4123. [CrossRef]

15. Barroso, G.; Li, Q.; Bordia, R.Q.; Motz, G. Polymeric and ceramic silicon-based coatings-A review. J. Mater. Chem. A 2019, 7, 1936-1963. [CrossRef] 
16. Kim, K.; Ju, H.; Kim, J. Pyrolysis behavior of polysilazane and polysilazane-coated-boron nitride for high thermal conductive composite. Compos. Sci. Technol. 2017, 141, 1-7. [CrossRef]

17. Marcano, D.C.; Kosynkin, D.V.; Berlin, J.M.; Sinitskii, A.; Sun, Z.; Slesarev, A.; Alemany, L.B.; Lu, W.; Tour, J.M. Improved synthesis of graphene oxide. ACS Nano 2010, 4, 4806-4814. [CrossRef]

18. McGrail, B.T.; Rodier, B.J.; Pentzer, E.B. Rapid functionalization of graphene oxide in water. Chem. Mater. 2014, 26, 5806-5811. [CrossRef]

19. Wie, J.; Kim, K.; Kim, J. High thermal conductivity composites obtained by novel surface treatment of boron nitride. Ceram. Int. 2020, 46, 17614-17620. [CrossRef]

20. Bauer, F.; Decker, U.; Dierdorf, A.; Ernst, H.; Heller, R.; Liebe, H.; Mehnert, R. Preparation of moisture curable polysilazane coatings. Prog. Org. Coat. 2005, 53, 183-190. [CrossRef]

21. David, L.; Bhandavat, R.; Barrera, U.; Singh, G. Silicon oxycarbide glass-graphene composite paper electrode for long-cycle lithium-ion batteries. Nat. Commun. 2016, 7, 10998. [CrossRef]

22. Socher, R.; Krause, B.; Muller, M.T.; Boldt, R.; Pötschke, P. The influence of matrix viscosity on MWCNT dispersion and electrical properties in different thermoplastic nanocomposites. Polymers 2012, 53, 495-504. [CrossRef]

23. Oh, H.; Kim, Y.; Wie, J.; Kim, K.; Kim, J. Tailoring of Si-C-N-O ceramic-coated reduced graphene oxide by oil/water-solution process for high thermal conductive epoxy composite with electrical insulation. Compos. Sci. Technol. 2020, 197, 108257. [CrossRef]

24. Bisht, A.; Dasgupta, K.; Lahiri, D. Investigating the role of 3D network of carbon nanofillers in improving the mechanical properties of carbon fiber epoxy laminated composite. Compos. Part A Appl. Sci. Manuf. 2019, 126, 105601. [CrossRef]

25. Renteria, J.D.; Ramirez, S.; Malekpour, H.; Alonso, B.; Centeno, A.; Zurutuza, A.; Cocemasov, A.I.; Nika, D.L.; Balandin, A.A. Strongly anisotropic thermal conductivity of free-standing reduced graphene oxide films annealed at high temperature. Adv. Funct. Mater. 2015, 25, 4664-4672. [CrossRef]

26. Oh, H.; Kim, J. Fabrication of polymethyl methacrylate composites with silanized boron nitride by in-situ polymerization for high thermal conductivity. Compos. Sci. Technol. 2019, 172, 153-162. [CrossRef]

27. Zhang, X.; Shen, L.; Wu, H.; Guo, S. Enhanced thermally conductivity and mechanical properties of polyethylene $(\mathrm{PE}) /$ boron nitride $(\mathrm{BN})$ composites through multistage stretching extrusion. Compos. Sci. Technol. 2013, 89, 24-28. [CrossRef]

28. Yang, S.; Li, W.-Z.; Bai, S.; Wang, Q. High-performance thermal and electrical conductive composites from multilayer plastic packaging waste and expanded graphite. J. Mater. Chem. C 2018, 6, 11209-11218. [CrossRef]

29. Goldin, N.; Dodiuk, H.; Lewitus, D. Enhanced thermal conductivity of photopolymerizable composites using surface modified hexagonal boron nitride fillers. Compos. Sci. Technol. 2017, 152, 36-45. [CrossRef]

30. Chee, S.S.; Jawaid, M.; Sultan, M.; Alothman, O.Y.; Abdullah, L.C. Thermomechanical and dynamic mechanical properties of bamboo/woven kenaf mat reinforced epoxy hybrid composites. Compos. Part B Eng. 2019, 163, 165-174. [CrossRef]

31. Joseph, S.; Appukuttan, S.P.; Kenny, J.M.; Puglia, D.; Thomas, S.; Joseph, K. Dynamic mechanical properties of oil palm microfibril-reinforced natural rubber composites. J. Appl. Polym. Sci. 2010, 117, 1298-1308. [CrossRef]

32. Zhang, X.; Alloul, O.; He, Q.; Zhu, J.; Verde, M.J.; Li, Y.; Wei, S.; Guo, Z. Strengthened magnetic epoxy nanocomposites with protruding nanoparticles on the graphene nanosheets. Polymers 2013, 54, 3594-3604. [CrossRef]

Publisher's Note: MDPI stays neutral with regard to jurisdictional claims in published maps and institutional affiliations.

(C) 2020 by the authors. Licensee MDPI, Basel, Switzerland. This article is an open access article distributed under the terms and conditions of the Creative Commons Attribution (CC BY) license (http://creativecommons.org/licenses/by/4.0/). 